\title{
A Comparative Structural, Optical and Electrical Properties of Metal-Semiconductor- Metal Based on Inalas /Ingaas
}

\author{
Benzeghda Sabah ${ }^{1 *}$, Bouhadiche Adil ${ }^{2}$, Benghorieb Soulef ${ }^{2}$, \\ Irma saraswati ${ }^{3}, \mathrm{~F}$ Hobar $^{1}$ \\ ${ }^{2}$ Research Unit Optics (UROP) Center for development of advanced Technologies (CDTA) University of Setif \\ ${ }^{3}$ Electrical Department Faculty of Engineering Univerfitas Sultan Ageng Tirtasa Banten
}

${ }^{1}$ Microsystems and Instrumentation Laboratory Department of Electronics

Faculty of Engineering Sciences University Mentouri Constantine, Algeria

*Corresponding author. Email: benzeghdasabah@gmail.com

\begin{abstract}
Interdigitated metal-semiconductor-metal (MSM) photodetectors based on InAlAs/InGaAs materials have attracted much attention for applications in high-speed optical-fiber communication systems which need efficient light detection in the 1.3-1.55 $\mu \mathrm{m}$ range [1]. The performance of thin-film inverted MSM are analyzed and compared to conventional MSM, that was modeled and simulated using Silvaco TCAD (Technology Computer Aided Design) and ATLAS software. We show how the interdigital spacing and thickness of the InGaAs layer influence the impulse response and the associated bandwidth [2].
\end{abstract}

Keywords: Metal-Semiconductor, Optical Properties, Metal Based Inalas

\section{INTRODUCTION}

The worldwide diffusion of optical fibers in communication for long distances, local area networks, has powered an increasing effort towards the realization of high speed and high efficiency optical detectors which require efficient light detection for wavelengths around 1.3-1.55 $\mu \mathrm{m}$ [3]. Interdigitated metal-semiconductormetal (MSM) structure is shown in Figure 1. It is a planar photodiode based on simple technology; it is easy to integrate [4].

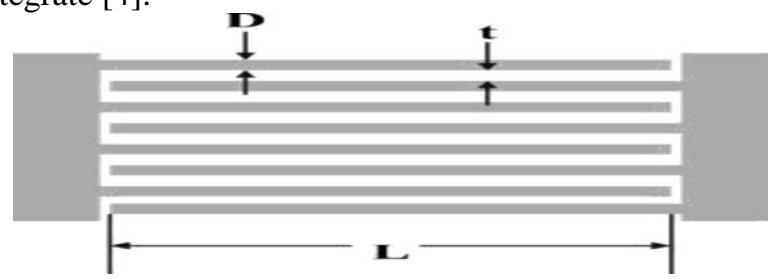

Figure 1. MSM photodetector with its typical interdigitated structure.

It consists of two Schottky electrodes, frequently interlinked in the form of a comb structure, leaving a free semiconductor surface between the two contacts which forms the active region in which light will be absorbed [4]. The application of a bias to the metallic fingers creates an electric field within the underlying semiconductor which acts to sweep the photogenerated carriers out of the device [5].

The main advantages of MSM PDs being the low capacitance, dark current and short impulse response. InGaAs based receivers have been under intensive investigation for long distance optical communication in the 1.3-1.55 $\mu \mathrm{m}$ bands because of their small bandgaps [6]. A thin high-bandgap material, such as AlInAs, between the InGaAs and the metal is required in order to reduce the dark current to reasonable levels [7].

In this paper, we analyze the performances of InAlAs/InGaAs Inverted- MSM PDs (I-MSM PDs), which are thin film MSM PDs with the fingers on the bottom of the device, and compare to conventional MSM PDs (C-MSM PDs) [8]. Device simulations are performed using Silvaco's 3-D ATLAS device simulation environment. 


\section{DEVICE STRUCTURE AND MODELS}

For the InP-based InGaAs material generally used in the optical communication band, the main obstacle of the metal-semiconductor system is its low Schottky barrier height $(-0.2 \mathrm{eV})$ [1], [9]. To solve this problem, a suitable barrier enhancement material must be introduced between the InGaAs light absorption layer and the metal electrodes [9].

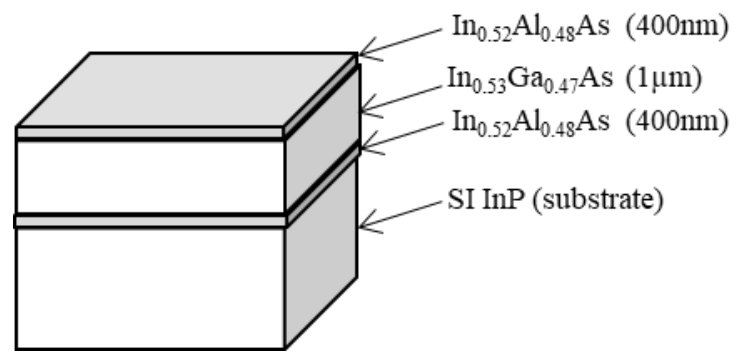

Figure 2. Device Structure.

Both I-MSM's and W-MSM It's consist of the same double structure grown lattice on a (100) SI InP (substrate)-In0.52Al0.48As (400nm)-In0.53Ga0.47As $(1 \mu \mathrm{m})-\mathrm{In} 0.52 \mathrm{Al} 0.48 \mathrm{As}(400 \mathrm{~nm})$, with all layers nominally undoped [1]. Fingers of Ti-Au $1 \mu \mathrm{m}$ wide separated by $1 \mu \mathrm{m}$ were defined on the $50 \mu \mathrm{m}$ diameter MSM's.

The simulated device is a three-dimensional structure as shown in Figure. 3. Device simulations are performed using Silvaco's 3-D ATLAS device simulation environment that simulates the optical, electrical, and thermal behavior of semiconductor devices. It solves the fundamental physical equations describing the dynamics of carriers in semiconductor devices and predicts terminal characteristics of semiconductor devices for steady state, transient and small signal AC simulation [9]. This model consists of a set of fundamental equations which link together the electrostatic potential and the carrier densities, within some simulation domain. These equations, which are solved, have been derived from Maxwell's laws and consist of Poisson's Equation (equation 1), which correlates the electrostatic potential to charge densities. The continuity equations (equation 2) keeps track of all the carriers in terms of movement with the transport equations (equation (3), [11, 12].

$$
\operatorname{div}(\varepsilon \nabla \psi)=-\rho
$$

Where $\psi$ is the electrostatic potential, $\varepsilon$ is the local permittivity, and $\rho$ is the local space charge density.

$$
\begin{aligned}
& \frac{\partial \mathrm{n}}{\partial \mathrm{t}}=\frac{1}{\mathrm{q}} \operatorname{div} \overrightarrow{\mathrm{J}}_{\mathrm{n}}+\mathrm{G}_{\mathrm{n}}-\mathrm{R}_{\mathrm{n}} \\
& \frac{\partial \mathrm{p}}{\partial \mathrm{t}}=-\frac{1}{\mathrm{q}} \operatorname{div} \underset{\mathrm{J}_{\mathrm{p}}}{\rightarrow}+G_{\mathrm{p}}-\mathrm{R}_{\mathrm{p}}
\end{aligned}
$$

Where $\mathrm{n}$ and $\mathrm{p}$ are the electron and hole concentration, $\rightarrow$ and $\rightarrow \underset{\mathrm{J}_{\mathrm{p}}}{\rightarrow}$ are the electron and hole current densities, Gn and Gp are the generation rates for electrons and holes, $\mathrm{Rn}$ and $\mathrm{Rp}$ are the recombination rates for electrons and holes, and $\mathrm{q}$ is the magnitude of the charge on an electron.

In this case, the current densities are expressed in terms of the quasi-Fermi levels $\varphi n$ and $\varphi p$ as:

$$
\begin{aligned}
& \overrightarrow{\mathrm{J}_{\mathrm{n}}}=-\mathrm{q} \mu_{\mathrm{n}} \mathrm{n} \nabla \varphi_{\mathrm{n}} \\
& \underset{\mathrm{J}_{\mathrm{p}}}{=}=-\mathrm{q} \mu_{\mathrm{p}} \mathrm{p} \nabla \varphi_{\mathrm{p}}
\end{aligned}
$$

Where $\mu \mathrm{n}$ and $\mu \mathrm{p}$ are the electron and hole mobilities. The first thing that needs to be specified is the mesh on which the device will be constructed (Figure. 3). ATLAS uses triangular meshes. The used materials require a different set of parameters to be specified or can be selected from a library that includes a number of common elements. These properties are defined by region.

After we defined doping profiles and structure meshing, we choose ATLAS environment physical model [11].

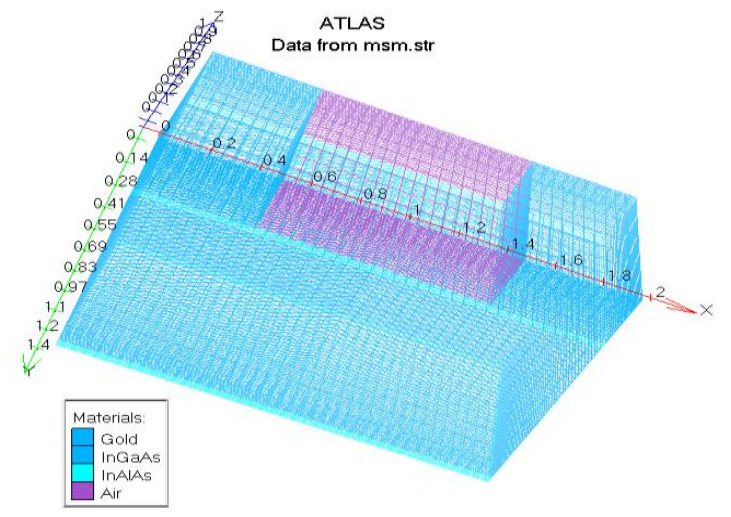

Figure 3. A Generated finer mesh structure of the 3D model.

An optical beam is adapted as a collimated source which is vertical illumination from the top for C-MSM and from the bottom for I-MSM. The beam is automatically split into a series of rays such that the sum of the rays covers the entire width of the illumination window.

Rays are also split at interfaces between regions into a transmitted ray and a reflected ray [11].

The influence of the applied bias with illuminated condition, the opto-generation rate must be calculated by integration of the Generation Rate Formula:

$$
G=\eta_{0} \frac{P \lambda}{h c} \alpha e^{-\alpha y}
$$

Where: 
$P$ contains the cumulative effects of reflections, transmissions, and loss due to absorption over the ray path. ๆo is the internal quantum efficiency which represents the number of carrier pairs generated per photon observed.

$\mathrm{y}$ is a relative distance for the ray in question.

$\mathrm{h}$ is Planck's constant

$\lambda$ is the wavelength.

$\mathrm{c}$ is the speed of light

$\alpha$ is is the absorption coefficient.

Light incident on the surface of the MSM structure is absorbed within the underlying semiconductor resulting in the creation of electron-hole-pairs [4].

The choice of numerical technique in solving equations of semiconductors can strongly affect both the convergence and CPU time required to complete a simulation run.

\section{RESULTS AND DISCUSSION}

The dark current is the leakage current when the photodetector is under bias but not exposed to the light source. The basic transport processes for a Schottky diode with n-type semiconductor under reverse bias are (1) emission of electrons from the semiconductor over the potential barrier into the metal, (2) quantum mechanical tunneling of electrons through the barrier, (3) recombination in the space-charge region, (4) diffusion of electrons in the depletion region, and (5) holes injected from the metal that diffuse into the semiconductor, (6) hopping of carriers between localized, defect related states, (7) tunneling to and from trap states within the bandgap [12] [13].

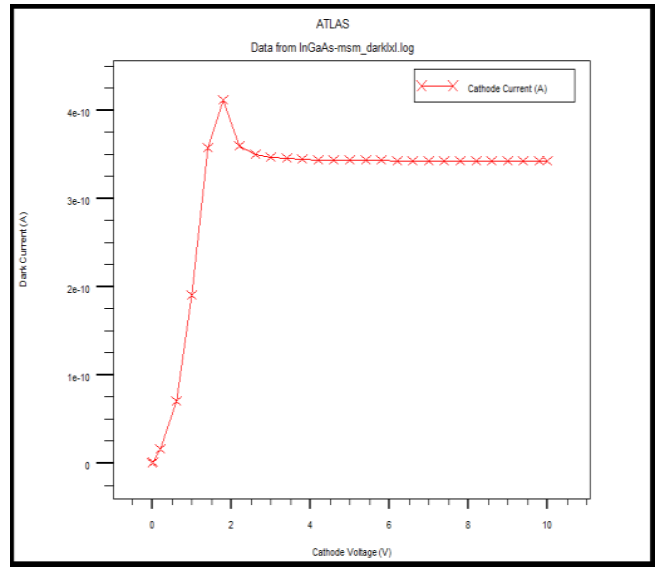

Figure 4. Dark current of the photo-detector MetalSemiconductor-Metal.

In Figure. 4 we give the dark current for a $1-\mu \mathrm{m}$ InGaAs detector with an interdigital spacing of $1 \mu \mathrm{m}$. The dark current is substantially independent of the bias applied to the photodiode. Both the W-MSM's and IMSM exhibited low dark current, for both devices it was less than $0.4 \mathrm{nA}$ for applied biases up to $10 \mathrm{~V}$, and the breakdown voltages were between 15 and $20 \mathrm{~V}$.

Both types of MSM's were optically excited at $1.3 \mu \mathrm{m}$ by a Gaussian pulse, the transient illumination with 10 ps rise time. The photoresponse is a strong function of speed of the devices. The simulated transient current pulses shown in Figure. 5 and 6.

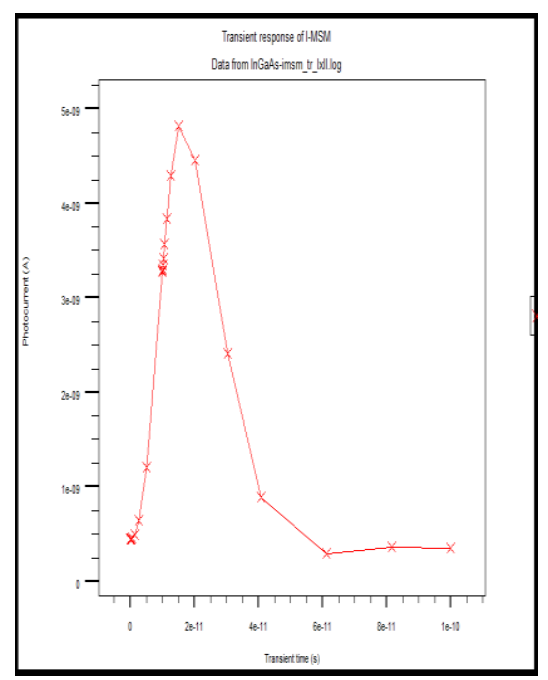

Figure 5. Transient response of I-MSM.

This tail is caused by carriers which pass deep into the InGaAs and the carrier collection times are consequently limited. Fig. 6 shows, then, that for short carrier collection times-which means a fast pulse response-the InGaAs layer must be thin enough to eliminate the longer carrier paths which loop down into the material.

The I-MSMs have slower response than W-MSM because carriers are created underneath the electrodes, thus resulting in longer collection time. FWHM=35ps and $20 \mathrm{ps}$ and rise time is $16 \mathrm{ps}$ and $15 \mathrm{ps}$ for I-MSM and W-MSM respectively, the Figure. 5 shows that long tail with heavier holes moving more slowly than electrons. As a result, the electrons quickly collect at the contacts, since they drift with high velocities both in the high-field region near the surface and in the low-field region in the bulk of the diode. Unlike holes, whose drift velocity drops continuously as the field decreases (Figure. 7), the electrons acquire a maximal drift velocity in a relatively low field. 


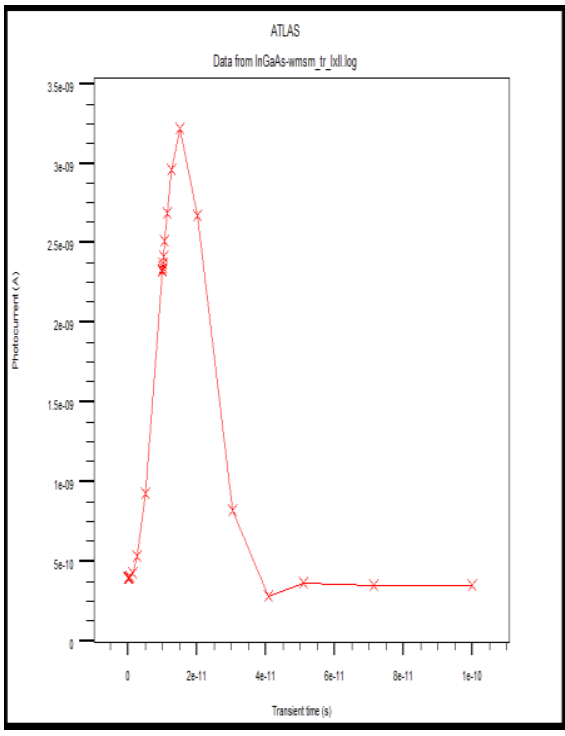

Figure 6. Transient response of W-MSM.

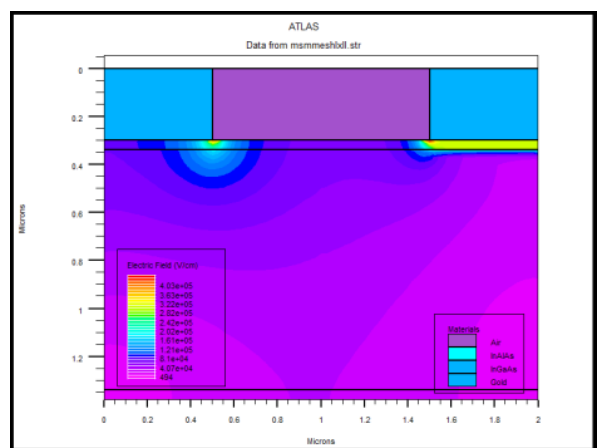

Figure 7. Distribution of electrical field in I-MSM.

For I-MSM and W-MSM (Figure. 8 and 9), simulation shows that after all electrons have left the active region, many holes still remain inside the diode, causing an extended tail in the impulse response of planar MSM diode and reducing the quantum efficiency.

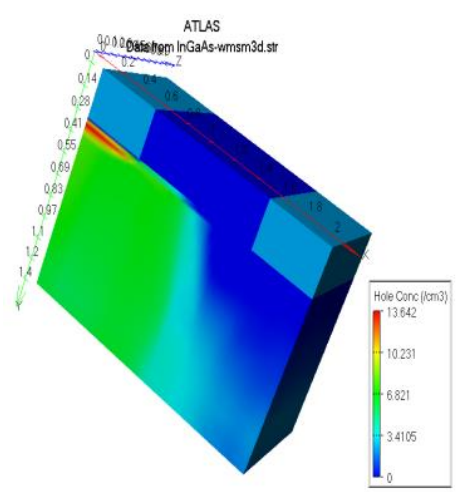

Figure 8. Hole concentration of W-MSM.

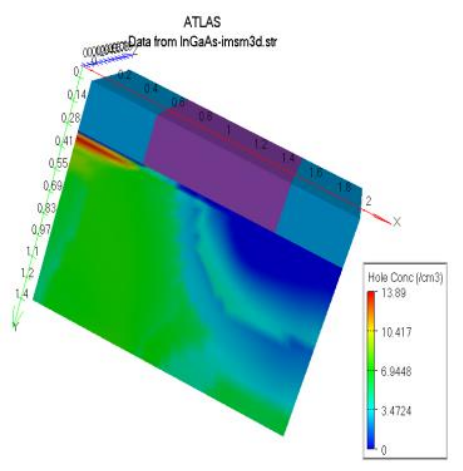

Figure 9. Hole concentration of I-MSM.

\section{CONCLUSION}

The main objective of this research was to simulate the photodetector in three dimensional, and develop process and device technologies that directly affect the performance of photoconductive photodetector used in high power microwave generation. The Silvaco suite was extensively used in this research on semiconductor devices.

Inverted MSM have been simulated to demonstrate that's a high quantum efficiency device with fast transient response. These I-MSM is compared with W-MSM and it is found that the responsivity of the I-MSM is larger than the W-MSM. This W-MSM is attractive photodetector for high speed integrated commuications applications with alignement tolerance due to the large size of these detectors relative to $\mathrm{p}-\mathrm{i}-\mathrm{n}$ detectors with the same capacitance [1].

\section{ACKNOWLEDGMENT}

The authors would like to thank prof D. Decoster, O. Vendier for helpful. This work was supported in part by, O. Vendier.

\section{REFERENCES}

[1] O. Vendier, Thin-Film Inverted MSM Photodetectors IEEE Transactions on Automatic Control, Vol. 53(Issue 1):257-272, February 2008.

[2] J.B.D. Soole; H. Schumacher, Transit-time limited frequency response of InGaAs MSM photodetectors, IEEE Transactions on Electron Devices, Volume: 37, Issue: 11, Nov 1990.

[3] R. Calarco_, M. Fiordelisi, Near-infrared metal_semiconductor_metal photodetector integrated on silicon, Thin Solid Films 391 Ž2001. 138_142.

[4] Didier Decoster, Joseph Harari, Optoelectronic Sensors, (Metal-Semiconductor-Metal Photodiodes, 2009, pp. 155-156). 
[5] Jianjun Gao, Optoelectronic Integrated Circuit Design and Device Modeling, John Wiley \& Sons, 19 sept. 201.

[6] Andrew Xiang, Walter Wohlmuth, Modelingof InGaAs MSM Photodetector for circuit-Level Simulation, Journal of lightwave technology, vol. 14, NO. 5, May 1996.

[7] S. V. Averine, Impulse Response of a Heterojunction MSM Photodiode, Technical Physics, Vol. 49, No. 6, 2004.

[8] Cheolung Cha, Jae Hong Kim, Improved Measurement-based Modeling of Inverted-MSM Photodetectors using On-wafer Calibration Structures, SPIE Vol. 5353, WA, 2004.

[9] YG Zhang, AZ Li, JX Chen, Improved performance of InAlAs-InGaAs-InP MSM photodetectors with graded superlattice structure grown by gas source MBE, IEEE Photonics Technology Letters, Volume: 8, Issue: 6, June 1996.

[10] Hong-Wei Lee, Solid State impact-Ionization Multiplier, Ph.D. dissertation, Dept. Elect. Computer Eng. Brigham Young Univ., Cambridge, MA, 1993.

[11] SILVACO, Atlas user's manual, device simulation software, 2002.

[12] S.M., Physics of semiconductor devices, John Wiley \& Sons Ltd., New York.

[13] Maximilien Billet, Sara Bretin, Fuanki Bavedila, InAlAs/InGaAs-MSM photodetectors based on optical cavity using metallic mirrors: $\mathrm{THz}$ frequency operation, high quantum efficiency and high saturation current, Appl. Phys. Lett. 114, 161104 (2019). 